\title{
Functional analysis of the -2548G/A leptin gene polymorphism in breast cancer cells
}

\author{
Marianna Terrasi $^{1,2}$, Elena Fiorio ${ }^{1,3}$, Anna Mercanti ${ }^{1,3}$, Mariusz Koda ${ }^{4}$, Camilo A. Moncada ${ }^{5}$, Stanislaw Sulkowski ${ }^{4}$, \\ Salim Merali ${ }^{5}$, Antonio Russo ${ }^{2}$ and Eva Surmacz ${ }^{*}$ \\ ${ }^{1}$ Sbarro Institute for Cancer Research and Molecular Medicine, Temple University, Philadelphia, PA \\ ${ }^{2}$ Section of Medical Oncology, Department of Surgical and Oncology, University of Palermo, Palermo, Italy \\ ${ }^{3}$ Department of Oncology, University of Verona, Verona, Italy \\ ${ }^{4}$ Department of Pathomorphology, Medical University of Bialystok, Bialystok, Poland \\ ${ }^{5}$ Department of Biochemistry School of Medicine, Temple University, Philadelphia, PA
}

Leptin is overexpressed in human breast tumors and is produced by breast cancer cells in response to obesity-related stimuli. The leptin promoter polymorphism Lep-2548G/A can be associated with increased leptin secretion by adipocytes and elevated cancer risk. However, molecular mechanisms underlying the link between Lep-2548G/A and breast cancer have never been addressed. Lep-2548G/A is proximal to a binding site for the transcriptional factor Sp1. Furthermore nucleolin, a transcriptional repressor, can bind $\mathrm{Sp} 1$ or its consensus site. Consequently, we focused on the impact of Lep-2548G/A on Sp1- and nucleolindependent leptin transcription in breast cancer cells. The Lep2548G/A was identified in a homozygous conformation in BT-474 and SK-BR-3 breast cancer cells, in a heterozygous conformation in MDA-MB-231 cells, and a wild-type Lep-2548G/G sequence was present in MCF-7 and ZR-75-1 cells. The occurrence of Lep2548A/A and Lep-2548G/A coincided with high and intermediate leptin mRNA expression, respectively, while cells containing Lep2548G/G expressed low leptin mRNA levels. We demonstrated that the existence of Lep-2548G/A improved efficient recruitment of Sp1 to DNA under insulin treatment, while Sp1 loading on DNA containing Lep-2548G/G was not insulin-dependent. In contrast, nucleolin binding to Lep-2548G/A was downregulated in response to insulin, while it was not regulated on Lep-2548G/G. The presence of Lep-2548G/A was studied in breast cancer epithelial cells by IHC and LCM. Interestingly, all 14 tumors expressing high leptin levels contained Lep-2548A/A. In conclusion, the occurrence of Lep-2548G/A can enhance leptin expression in breast cancer cells via Sp1- and nucleolin-dependent mechanisms and possibly contribute to intratumoral leptin overexpression. (C) 2009 UICC

Key words: leptin; polymorphism; breast cancer; insulin; Sp1; nucleolin

Leptin is an adipose tissue-derived hormone whose main role is to control appetite and energy balance by acting in the hypothalamus. Leptin levels are proportional to fat body mass and are higher in women than in man. ${ }^{1}$ In addition, leptin has been shown to modulate several other processes, such as immune response, fertility, hematopoiesis. ${ }^{1}$ In peripheral organs, leptin can act as a mitogenic, angiogenic, and survival factor. ${ }^{2}$ Recent studies in cellular and animal models provided strong evidence that leptin can be involved in neoplastic processes, for instance, in the development and progression of breast, colorectal, and endometrial cancers. $^{3-5}$ In addition, both leptin and its receptor (ObR) have been detected in different neoplastic tissues, usually at levels higher than that in respective normal tissues. ${ }^{6-8}$ Interestingly, overexpression of the leptin system in breast cancer appears to be associated with higher tumor grade and size. ${ }^{9-13}$ The mechanisms of leptin overexpression in breast cancer cells is partially known, for instance, we have shown that leptin transcription and synthesis can be induced by hypoxia or high levels of insulin. ${ }^{9,13,14}$

Here we investigated whether leptin expression in breast cancer cells could be related to the existence of the Lep-2548G/A polymorphism in the promoter region of leptin gene.

Lep-2548G/A has previously been shown to correlate with variations in serum leptin levels, degree of obesity, as well as cancer. ${ }^{15-17}$ In particular, studies of Mammes et al. using DNA from peripheral blood cells documented that the Lep-2548G/A polymorphism was associated with higher leptin levels independent of BMI, before and after diet. ${ }^{15}$ In addition, male, but not female, subjects with the Lep-2548A/A genotype had higher leptin concentrations adjusted for fat mass. ${ }^{16}$ Similar studies suggested that nonobese women with Lep-2548A/A had higher serum leptin levels adjusted for body mass index, and significantly higher adipose tissue leptin mRNA expression than did carriers of the wildtype Lep-2548G/G sequence. ${ }^{17}$

In the context of neoplastic processes, Lep-2548A/A detected in DNA from peripheral blood leucocytes associated with increased risk for non-Hodgkin lymphoma, ${ }^{18}$ and both Lep-2548A/A and Lep-2548G/A with non small cell lung cancer as well as prostate and breast carcinoma. ${ }^{19,20}$ Furthermore, the existence of Lep2548A/A has been shown to correlate with significantly increased breast cancer risk, and the presence of Lep-2548G/A with intermediate risk, compared with the wild-type Lep-2548G/G genotype. ${ }^{21}$ The Lep-2548G/A polymorphism also correlated with a larger breast tumor size at diagnosis and a shorter disease-free survival of patients. ${ }^{21}$ However, the functional significance of the Lep$2548 \mathrm{G} / \mathrm{A}$ polymorphism and its occurrence in cancer tissues has never been experimentally addressed, and is a subject of this study.

The human leptin gene promoter $(\sim 2,500 \mathrm{bp})$ contains several consensus binding sites for a variety of transcription factors. ${ }^{22}$ The Lep-2548 does not map to any known regulatory site, but is proximal to an Sp1 binding motif (CCCGCCT; -2539/-2534) that is known to be regulated by insulin in breast cancer cells. ${ }^{23}$ Nucleolin is another nuclear factor that can bind a Sp1 consensus site in the promoter of cytosolic phospholipase $\mathrm{A}_{2}\left(\mathrm{CPLA}_{2}\right)^{24}$ and that can interact with $\mathrm{Sp}$, as reported for human keratinocyte (HaCat) cell extracts. ${ }^{25}$

Nucleolin is a multifunctional phosphoprotein involved in ribosomal RNA transcription, maturation and assembly, ${ }^{26}$ chromatin decondensation ${ }^{27}$ as well as transcriptional regulation, ${ }^{28}$ including modulation of Sp1-dependent gene expression. ${ }^{24}$ Furthermore, insulin may induce changes in the phosphorilation of nucleolin. ${ }^{29}$

Consequently, we studied in breast cancer cell models if the occurrence of the Lep-2548G/A polymorphism can enhance basal and insulin-induced leptin expression via Sp1- and nucleolin-dependent mechanisms. In addition, the existence of the Lep-2548G/ A polymorphism was assessed in breast cancer tissues expressing high leptin levels using laser capture microdissection.

Grant sponsors: W.W. Smith Charitable Trust, Sbarro Health Research Organization.

* Correspondence to: Sbarro Institute for Cancer Research and Molecular Medicine, Temple University, 1900 N 12th Street, BioLife Bldg 446, Philadelphia, PA 19122, USA. E-mail: surmacz@temple.edu

Received 17 October 2008; Accepted after revision 6 February 2009

DOI 10.1002/ijc. 24372

Published online 18 February 2009 in Wiley InterScience (www.interscience. wiley.com). 


\section{Material and methods}

\section{Cell culture}

MCF-7, MDA-MB-231, SK-BR-3, BT-474, ZR-75-1 cell lines were purchased from the American Type Culture Collection (Rockville, MD). MCF-7 and MDA-MB-231 cells were grown in Dulbecco's modified Eagle's medium (DMEM:F12) (Cellgro, Herndon, VA) containing 5\% calf serum and 1\% Penicillin/Streptomycin (P/S) (Cellgro). SK-BR-3 cells were grown in DMEM:F12 containing 10\% fetal bovine serum (FBS) and $1 \% \mathrm{P} / \mathrm{S}$. BT-474 cells were grown in DMEM:F12 containing 10\% FBS, $1 \% \mathrm{P} / \mathrm{S}$ and $0.01 \mu \mathrm{g} / \mathrm{ml}$ insulin. ZR-75-1 cells were grown in RPMI-1640 (BioWhittaker, Walkersville, MD) containing 10\% FBS, $1 \% \mathrm{P} / \mathrm{S}$ and $1 \mathrm{mM}$ sodium pyruvate (Cellgro). SK-BR-3 AND ZR-75-1 cell lines were omitted from experiments requiring serum-free medium (SFM) synchronization because serum depletion induced loss of adhesion and anoikis in these cells.

\section{Detection of the Lep-2548G/A polymorphism}

Genomic DNA was extracted from breast cancer cells with DNeasy Tissue Kit (Qiagen, Hilden, Germany). The existence of the Lep-2548G/A polymorphism was studied by PCR using a 2700 thermal cycler (Applied Biosystems, Foster City, CA). The following primers were used: forward $5^{\prime}$-ACTTTATAGACACA GAAATGAAAA- $3^{\prime}$ reverse $5^{\prime}$-AAAATTTATGTTCCTCTGC CAC- $3^{\prime}$. The PCR conditions were: 25 cycles at $30 \mathrm{sec}$ at $94^{\circ} \mathrm{C}$, $30 \mathrm{sec}$ at $53^{\circ} \mathrm{C}, 30 \mathrm{sec}$ at $72^{\circ} \mathrm{C}$. The quality of PCR products was evaluated in a $2 \%$ agarose gel electrophoresis stained with ethidium bromide. Direct sequencing of the PCR products was performed using a BigDye Terminator reaction chemistry on ABI PRISM 377 Genetic Analyzer (Applied Biosystems). Each genetic variant was confirmed by direct sequencing of at least two independent samples.

\section{Western blotting $(W B)$}

Eighty percent confluent cultures were transferred to phenol red-free SFM for $24 \mathrm{hr}$ (MCF-7 and MDA-MB-231 cells) or $72 \mathrm{hr}$ (BT-474) and then stimulated with $2 \mu \mathrm{g} / \mathrm{ml}$ insulin for $3 \mathrm{~h}$, or left untreated. Because normal growth medium for BT-474 cells contains insulin, this cell line was cultured in SFM for $72 \mathrm{hr}$ to completely eliminate insulin effects on gene transcription, including Sp1-mediated regulation. ${ }^{23}$ Then cells were lysed to separate cytoplasmic and nuclear proteins, as described before. ${ }^{30}$ The expression of nuclear proteins was analyzed in $200 \mu \mathrm{g}$ of nuclear cell lysates. The following antibodies (Abs) were used for WB: Sp1 (PEP2) polyclonal (p)Ab (Santa Cruz Biotechnology, CA), nucleolin mAb (Santa Cruz) and c-Jun (N) polyclonal (Santa Cruz). The proteins were separated on a $4-15 \%$ polyacrylamide gel, immunoblotted and quantified, as described before. ${ }^{30}$

\section{Chromatin immunoprecipitation (ChIP)}

We followed ChIP methodology described in detail previously. ${ }^{31}$ Eighty percent confluent cultures were transferred to SFM for $24 \mathrm{hr}$ (MCF-7 and MDA-MB-231 cells) or $72 \mathrm{~h}$ (BT-474) and then stimulated with $2 \mu \mathrm{g} / \mathrm{ml}$ insulin for $3 \mathrm{hr}$, or left untreated. Following treatment, the cells were crosslinked with $1 \%$ formaldehyde and chromatin was collected and sonicated. Soluble chromatin was immunoprecipitated with the following Abs: Sp1 $\mathrm{PEP}_{2}$ (Santa Cruz Biotechnology), nucleolin C23 MS-3 (Santa Cruz). DNA-protein immune complexes were eluted, reverse crosslinked and DNA was extracted. The binding of Sp1, nucleolin to the leptin promoter region -2632/-2453 containing the -2548 site was tested using the following primers: forward $5^{\prime}$ ACTTTATAGACA CAGAAATGAAAA and reverse 5'-CTCGGCACCGTCTCCT TG- $3^{\prime}$. The PCR conditions were: 30 cycles at $30 \mathrm{sec}$ at $94^{\circ} \mathrm{C}$, $30 \mathrm{sec}$ at $53^{\circ} \mathrm{C}, 30 \mathrm{sec}$ at $72^{\circ} \mathrm{C}$. In control samples, the primary Abs were replaced with appropriate nonimmune IgG.
DNA affinity precipitation assay (DAPA)

Binding of $\mathrm{Sp} 1$ and nucleolin to the leptin promoter region containing an Sp1 binding motif and Lep-2548 site was assessed in vitro using modified DAPA protocol, as described by us before. ${ }^{32}$ Briefly, nuclear extracts were obtained from cells stimulated or not with $2 \mu \mathrm{g} / \mathrm{ml}$ insulin for $3 \mathrm{hr}$. A total of $300 \mu \mathrm{g}$ of nuclear proteins were mixed with $2 \mu \mathrm{g}$ of specific biotinylated DNA probes (see below) in $400 \mu$ of Buffer D (20 mM HEPES pH 7.9, 10\% glycerol, $50 \mathrm{mM} \mathrm{KCl}, 0.2 \mathrm{mM}$ EDTA, $1.5 \mathrm{mM} \mathrm{MgCl} 2,10 \mathrm{mM}$ $\mathrm{ZnCl} 2,1 \mathrm{mM}$ DTT and $0.25 \%$ Triton X100) and then incubated on ice for $45 \mathrm{~min}$. After that, $50 \mu \mathrm{l}$ of streptavidin beads (Pierce, Rockford, IL) were added and the samples were agitated for $2 \mathrm{hr}$ at $4^{\circ} \mathrm{C}$. Next, the agarose beads-protein complexes were collected by brief centrifugation and washed twice in Buffer D. Proteins were uncoupled from DNA probes by addition of $40 \mu \mathrm{l}$ SDS loading buffer, and heating at $96^{\circ} \mathrm{C}$ for $10 \mathrm{~min}$. After removal of the beads, the supernatants were analyzed by WB for the presence of Sp1 and nucleolin. The specific DNA probes were prepared by annealing a biotinylated sense oligonucleotide (for $-2548 \mathrm{G} / \mathrm{G}$ : $5^{\prime}$ Bio GGGTTGCGCTGATCCTCCCGCCTCAGTTCCCTA- ${ }^{\prime}$ and for -2548A/A: 5'-Bio GGGTTGCACTGATCCTCCCGCCTCAG TCTCCCTA- $3^{\prime}$ ) with nonbiotinylated antisense oligonucleotide (for -2548G/G: 5'-CCCAACGCGACTAGGAGGGCGGAGTCA GAGGGAT-3' and for -2548A/A: 5'-CCCAACGTGACTAGGA GGGCGGAGTCAGAGGGAT3'). Unlabeled probes were used as negative controls. As an additional control, a 10-fold excess of unlabeled probes was added to the nuclear lysates 30 min prior to the addition of the labeled probes to block specific probe-protein interactions.

\section{Real time-quantitative $P C R(Q-P C R)$}

The cells were synchronized in SFM for $24 \mathrm{hr}$ (MCF-7, MDAMB-231) or $72 \mathrm{hr}$ (BT-474) and then treated for $24 \mathrm{hr}$ with $2 \mu \mathrm{g} /$ $\mathrm{ml}$ insulin. Total cellular RNA was isolated using TRIzol reagent (Invitrogen, Carlsbad, CA). $5 \mu \mathrm{g}$ of total RNA was reverse transcribed using the TaqMan RT kit (Applied Biosystems, Branchburg, CA) according to vendor's instructions. $5 \mu \mathrm{l}$ of the RT products were used to amplify leptin sequences using the Hs00174877 A1 Lep TaqMan kit (Applied Biosystems). To normalize QRTPCR reactions, parallel TaqMan $\beta$-actin control reagents assays (Applied Biosystems) were run on each sample. Changes in the leptin mRNA content relative to $\beta$-actin mRNA were determined using a comparative CT method (ABI User Bulletin no. 2) to calculate changes in $\mathrm{CT}$, and ultimately fold and percent change. An average CT value for each RNA was obtained for triplicate reactions.

\section{Breast cancer patients and samples}

The analysis of leptin gene polymorphism was performed in 14 breast cancer tissue samples, previously identified as strongly leptin-positive. ${ }^{9}$ Tissue samples were obtained from women who underwent partial or total mastectomy for primary breast cancer. The age of patients ranged from 45 to 71 years. Histopathological examination of sections was based on the WHO and pTN classification of breast tumors. Tumor grading $(\mathrm{G})$ was identified as $\mathrm{G} 2$ in $78.5 \%$ and $\mathrm{G} 3$ in $21.5 \%$ of cases; node status $(\mathrm{N})$ was identified as $\mathrm{N} 1$ (cancer cells found in one lymph nodes) in $43 \%$ and negative (absence of regional metastases, N0) in $57 \%$ of cases. Tumor size $(\mathrm{pT})$ was identified as pT1 $(0-2 \mathrm{~cm})$ in $40 \%$ and pT2 (2-5 $\mathrm{cm}$ ) in $60 \%$ of cases. Immediately after excision, tissue samples were fixed in $10 \%$ buffered formaldehyde solution, embedded in paraffin blocks at $56^{\circ} \mathrm{C}$.

\section{Laser capture microdissection (LCM) of breast cancer tissue}

Sections of $6 \mu \mathrm{m}$ were cut from breast cancer tissue specimens paraffin-embedded and mounted on PEN membrane glass slides (Arcturus, Mountain View, CA). The expression of leptin was investigated by IHC with specific Abs. Tissue sections were deparaffinized in xylene and rehydrated in graded series of 


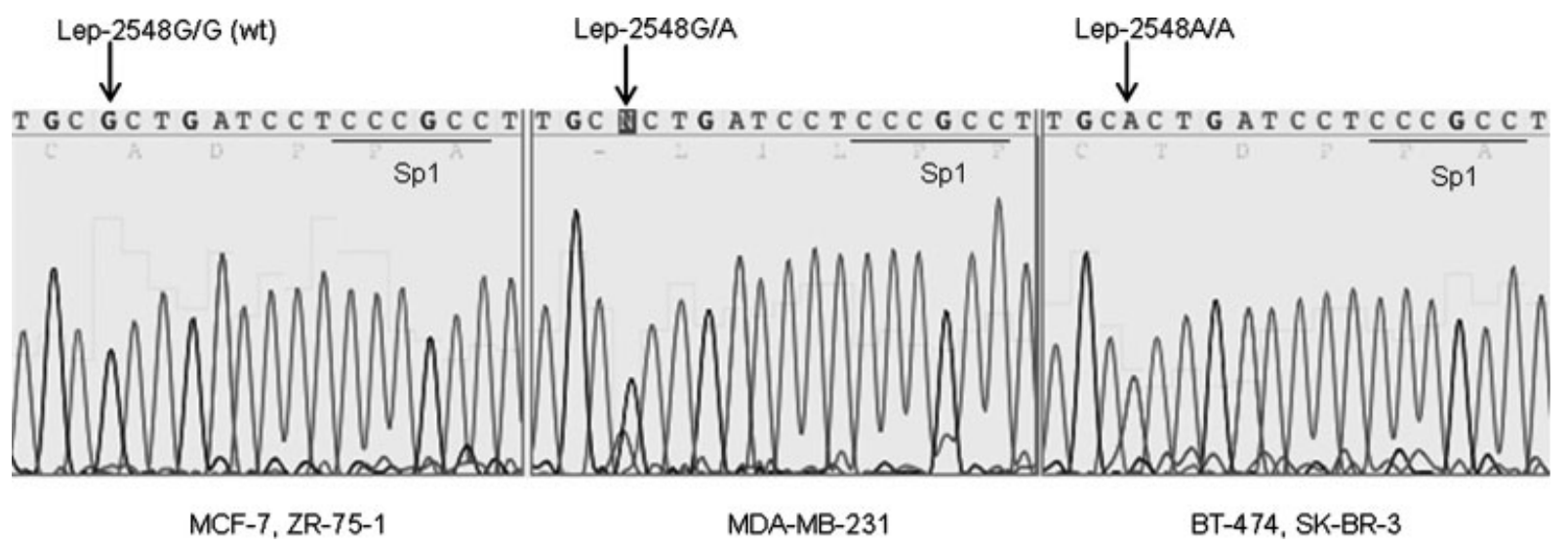

Figure 1 - Detection of the Lep-2548G/A polymorphism in breast cancer cells. Presence of the Lep-2548G/A polymorphism was assessed by direct sequencing in five different breast cancer cell lines: MCF-7 and ZR-75-1 cells displayed a wild type (Lep-2548G/G) genotype, MDAMB-231 cells were heterozygotic (Lep-2548G/A), and SK-BR-3 and BT-474 cells were homozygotic for the polymorphism (Lep-2548A/A).

ethanol. After rinsing in PBS, endogenous peroxidase activity was inhibited by incubation with $30 \%$ hydrogen peroxide, diluted in $100 \%$ methanol for $30 \mathrm{~min}$ at $4^{\circ} \mathrm{C}$. After three washes in PBS the sections were incubated with $1.5 \%$ blocking serum for $1 \mathrm{hr}$, then the sections were incubated for $3 \mathrm{hr}$ with primary antibodies. For leptin staining, we used the A20 leptin polyclonal Ab (pAb) (Santa Cruz) at 1:100 dilution, as described before. ${ }^{9}$ The leptin antigens were detected with avidin-biotin-peroxidase ABC staining systems (Santa Cruz). All slides were counterstained with Mayer's hematoxylin. Areas of interest were collected using a Veritas (Arcturus, Mountain View, CA) LCM instrument equipped with Veritas image archiving software. The cutting beam diameter was $2.0 \mu \mathrm{m}$ and the capture beam diameter was 7.0-9.0 $\mu \mathrm{m}$. The laser intensity control was set at $4.0 \mathrm{mV}$ for cutting and $200 \mathrm{mV}$ for capture. Typically, 6-8 laser pulses were required to cut out the selected area, which was then captured on thermoplastic film coated CapSure ${ }^{\mathrm{TM}}$ Macro Caps mounted films (Arcturus) according to manufacturer's directions. Portions of the films holding captured areas were placed in microcentrifuge tubes and used for DNA extraction performed with PicoPure DNA Extraction Kit (Arcturus). Detection of the Lep-2548G/A polymorphism was performed as described above.

\section{Results}

Detection of the Lep-2548G/A polymorphism in breast cancer cell lines

Genotypes were determined for different breast cancer cell lines to test if the occurrence of the Lep-2548G/A polymorphism in the leptin promoter can enhance basal and insulin-induced leptin expression. MCF-7 and ZR-75-1 cells contained the wild type (Lep-2548G/G) promoter sequence, MDA-MB-231 cells contained heterozygous (Lep-2548G/A) variant, while BT-474 and SK-BR-3 cells were homozygous for the (Lep-2548A/A) polymorphism (Fig. 1). MCF-7, MDA-MB-231 and BT-474 cells were selected for further experiments.

\section{Interaction between Sp1, nucleolin and the leptin promoter} domain containing the Lep-2548 site

The Lep-2548 site doesn't map to any known regulatory site, but is proximal to an Sp1 binding motif (CCCGCCT; -2539/ -2534). We recently reported that this leptin promoter region is regulated by $\mathrm{Sp} 1$ in an insulin-dependent fashion in MDA-MB231 cells. $^{23}$ Interestingly, a Sp1-binding sequences of the cytosolic phospholipase $\mathrm{A}_{2}$ promoter seem to be also recognized by another transcriptional regulator, nucleolin. ${ }^{24}$ It has also been proved a protein-protein interaction between $\mathrm{Sp} 1$ and nucleolin in human keratinocyte (HaCat) cell extracts. ${ }^{25}$ Here we investigated how the

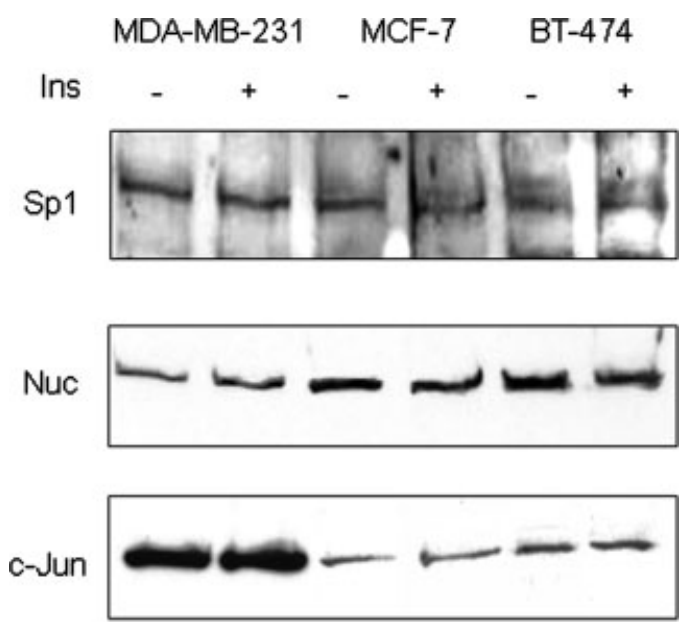

Figure 2 - Expression of Sp1 and nucleolin in breast cancer cell lines. The expression of nuclear Sp1 and nucleolin (Nuc) was analyzed by WB, as described in Material and methods section, in $200 \mu \mathrm{g}$ of nuclear proteins obtained from cells treated with $2 \mu \mathrm{g} / \mathrm{ml}$ insulin (Ins) for $3 \mathrm{hr}$, or left untreated. Protein loading was controlled by reprobing the WB filter for the expression of a nuclear marker c-Jun.

presence of the Lep-2548G/A polymorphism might affect insulindependent leptin transcription.

First, we determined basal and insulin-induced nuclear expression levels of $\mathrm{Sp} 1$ and nucleolin in different breast cancer cell lines (Fig. 2). The abundance of $\mathrm{Sp} 1$ and nucleolin in the nuclei of MCF-7, MDA-MB-231 and BT-474 cells varied depending on cell line, but in each case was not affected by insulin addition (Fig. 2).

Next, we investigated, by ChIP assays, the binding of Sp1 and nucleolin to the Lep-2548 region using native chromatin of MCF7, MDA-MB-231 and BT-474 cell lines (Fig. 3a). We found that, under insulin treatment, the presence of the polymorphism improves Sp1 binding to the promoter, while it reduces nucleolin association in MDA-MB-231 (Lep-2548G/A) and BT-474 (Lep2548A/A) cells. In MCF-7 (Lep-2548G/G) we did not observe any regulation of $\mathrm{Sp} 1$ or nucleolin under insulin treatment. Chromatin samples precipitated with control antibodies did not reveal any PCR products for Lep-2548 region (data not shown).

To specifically map Sp1 and nucleolin binding site within the ChiP-tested DNA region, we used DAPA protocol. Short biotinylated DNA probes containing a Sp1 binding motif adjusted to Lep$2548 \mathrm{~A} / \mathrm{A}$ or Lep-2548G/G sequences were hybridized with 
a

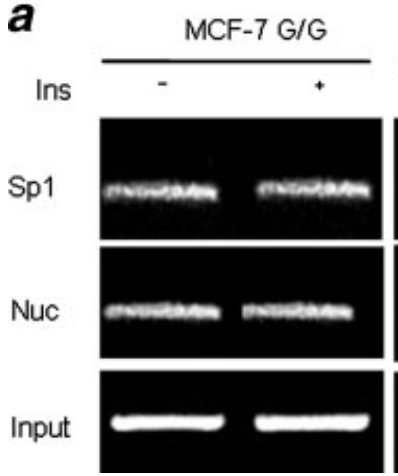

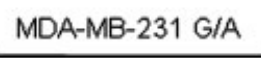

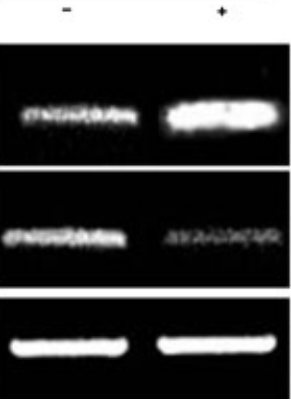

BT-474 AVA

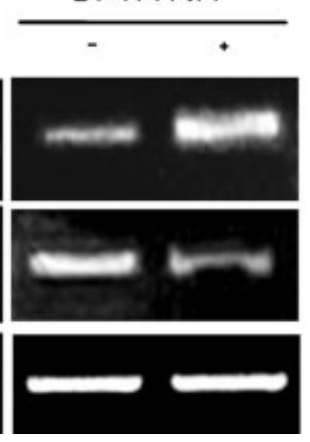

\section{b}

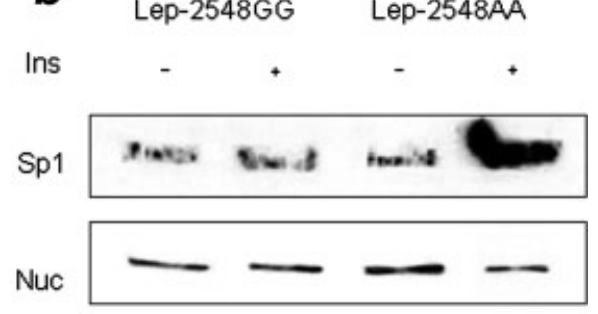

Figure 3 - Interaction between Sp1, nucleolin and the leptin promoter domain containing the Lep-2548 site. (a) The binding of Sp1 and nucleolin (Nuc) to the leptin promoter region containing an Sp1 binding motif and the Lep-2548 site was tested by ChIP, as described in Material and methods section. Cells were transferred to SFM for $24 \mathrm{hr}$ (MCF-7 and MDA-MB-231 cells) or $72 \mathrm{hr}$ (BT-474) and then stimulated with $2 \mu \mathrm{g} / \mathrm{ml}$ insulin for $3 \mathrm{hr}$. In control samples, the primary Abs were replaced with nonimmune IgG (not shown). (b) The binding of Sp1 and nucleolin (Nuc) to the leptin promoter region containing an Sp1 site and the Lep-2548 sequence was assessed in vitro using modified DAPA, as described in Material and methods section. MDA-MB-231 cells were transferred to SFM for $24 \mathrm{hr}$ and then stimulated with $2 \mu \mathrm{g} / \mathrm{ml}$ insulin for $3 \mathrm{hr}$. Next, $300 \mu \mathrm{g}$ of nuclear protein were mixed with $2 \mu \mathrm{g}$ of specific biotinylated DNA probes for Lep-2548G/G or Lep-2548A/A and nonbiotinylated antisense oligonucleotides, as detailed in Material and methods section. Control nonbiotinylated probes did not produce any Sp1 or nucleolin binding (not shown).

nuclear proteins obtained from MDA-MB-231 cells, stimulated or not with $2 \mu \mathrm{g} / \mathrm{ml}$ insulin. Confirming ChIP results, we found that the Lep-2548A/A genotype improves recruitment of Sp1 to DNA under insulin treatment, while it reduces the recruitment of nucleolin. In contrast, binding of nucleolin was not regulated on the wild type Lep-2548G/G (Fig. 3b). Unlabeled probes, used as controls, did not precipitated Sp1 or nucleolin proteins (data not shown).

\section{Differential leptin mRNA expression in breast cancer cells carrying different Lep-2548 genotypes}

To assess whether the presence of Lep-2548G/A and differential binding of $\mathrm{Sp} 1$ and nucleolin to this site can be associated with differential leptin transcription, we used QRT-PCR to measure basal and insulin-induced leptin mRNA levels in MCF-7, MDA-MB-231 and BT-474 cells. The lowest levels of basal leptin mRNA were found in MCF-7 cells (Lep-2548G/G), intermediate levels in MDA-MB-231 cells (Lep-2548G/A), and the highest levels in BT-474 cells (Lep-2548A/A) (Fig. 4). Treatment with $2 \mu \mathrm{g} /$ $\mathrm{ml}$ insulin did not significantly modulate leptin expression in MCF-7 cells (Lep-2548G/G), relative to basal expression, while enhanced leptin mRNA levels in MDA-MB-231 cells (Lep2548G/A). In BT-474 cells (Lep-2548A/A) expressing high basal leptin mRNA insulin addition did not significantly modulate leptin expression (Fig. 4).

\section{Detection of the Lep-2548G/A polymorphism in breast cancer tissues}

We performed preliminary analysis of leptin promoter polymorphism in human breast cancer biopsies. Leptin expression in the cancer tissues studied was previously identified as very high $(+++$ score $) .{ }^{9}$ In this analysis, we confirmed by specific IHC staining high leptin expression in the selected tumors. The leptinexpressing epithelial cells were isolated by laser microdissection (LCM). Analysis of DNA from the leptin overexpressing cancer tissues revealed the presence of the Lep-2548A/A polymorphism in all samples.

\section{Discussion}

The common Lep-2548G/A polymorphism in the leptin gene promoter has previously been associated with degree of obesity, variations in serum leptin levels, and leptin synthesis by adipose

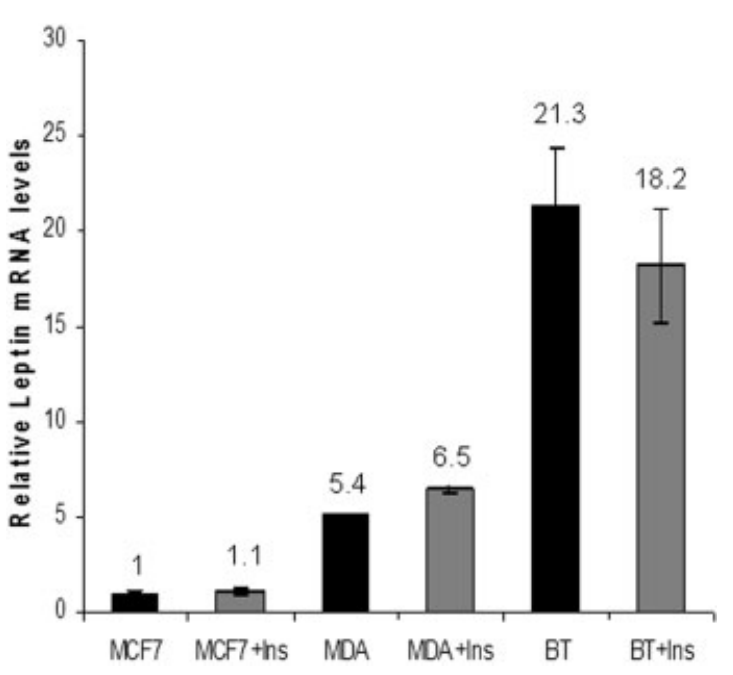

Figure 4 - Leptin mRNA expression in breast cancer cell lines with different genotype for the Lep-2548 polymorphism. The abundance of leptin mRNA was studied using QRT-PCR in MCF-7, MDAMB-231 (MDA) and BT-474 (BT) cells untreated or treated with $2 \mu \mathrm{g} / \mathrm{ml}$ insulin (Ins) for $24 \mathrm{hr}$, as described in Material and methods section. The graphs represent the increase of leptin mRNA relative to the increase of constitutive mRNA control ( $\beta$-actin) in the same sample \pm SDs. The differences between the two conditions (untreated and treated) were statistically significant $(p<0.05)$.

tissue. ${ }^{15}$ New data suggested the link between Lep-2548G/A and risk of neoplastic disease. ${ }^{18-21}$

Studies in cellular and animal models strongly suggested that high levels of leptin might promote breast carcinogenesis. ${ }^{3}$ Reports from our and other laboratories demonstrated that both leptin and ObR are overexpressed in breast cancer cells, $4,6,33$ which implies that leptin could promote breast cancer not only through endocrine but also through autocrine mechanisms. As reported by us before, leptin synthesis in breast cancer cells can be upregulated by obesity-related stimuli, such as high levels of insulin, insulin-like growth factor I, or estrogens. ${ }^{9,23,34}$ However, the possibility that leptin promoter poymorphism might influence leptin transcription in breast cancer cells have never been studied. 
Until now, the molecular basis of the link between the occurrence of Lep-2548G/A and increased leptin expression have not been investigated. One report implied, however, that some transcriptional events can be involved: electric mobility shift assay revealed that nuclear extracts from human monocyte U937 cells

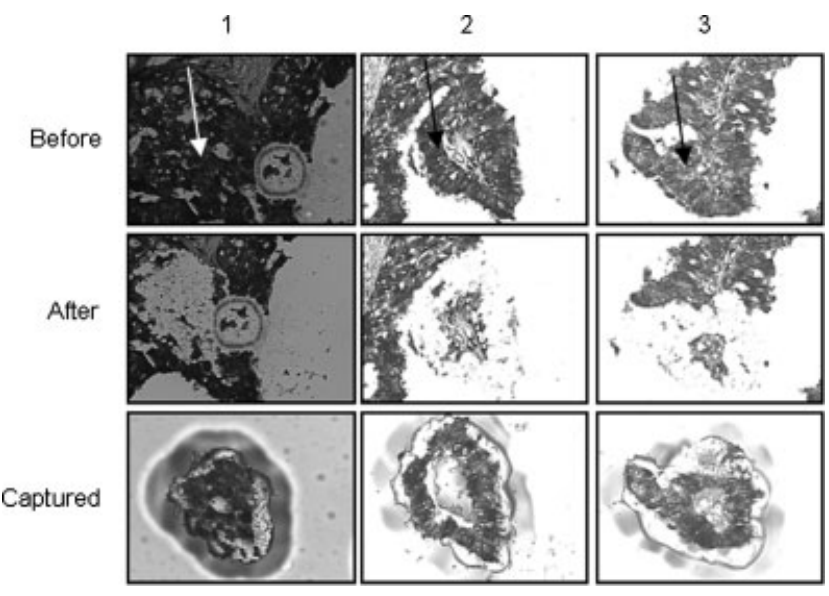

Figure 5 - Detection of the Lep-2548G/A polymorphism in breast cancer tissues. Before LCM experiment, the expression of leptin in each tissue sample was visualized by IHC with specific Abs and all slides were counterstained with Mayer's hematoxylin. The areas before and after capture, as well as captured areas were photographed using Veritas image archiving software. Three representative LCM experiments $(1,2,3)$ are shown. Arrows indicate leptinpositive areas. form a protein-DNA complex with the polymorphic site Lep2548G/A, binding with higher affinity to the Lep-2548A allele. ${ }^{17}$ However, using nuclear proteins from human subcutaneous adipocytes, two complexes were found, one with the same mobility as the protein complex derived from U937 cells and the other with higher mobility. The molecular composition of these two complexes has not been described. ${ }^{17}$

Here we investigated if human breast cancer cells contain the Lep-2548G/A polymorphism and if the presence of this variant can affect leptin gene expression. Because the Lep-2548G/A site in the leptin promoter is proximal to $\mathrm{Sp} 1$ binding motif ${ }^{23}$ and nucleolin could interact with $\mathrm{Sp}^{25}$ and bind his consensus sites, ${ }^{24}$ we focused on the interplay between Lep-2548G/A and the two transcriptional regulators in the process of basal and insulininduced leptin mRNA synthesis.

We first identified three different Lep-2548 genotypes in breast cancer cell lines. Specifically, MCF-7 and ZR-75-1 cells contained the Lep-2548G/G wild-type genotype, while MDA-MB-231 contained the Lep-2548G/A polymorphism, and BT-474 and SK-BR3 cells expressed the Lep-2548A/A genotype. ChIP assays suggested that in MCF-7 cells, basal binding of Sp1 or nucleolin to Lep-2548G/G was not affected by insulin treatment. On the other hand, in MDA-MB-231 and BT-474 cells, insulin induced Sp1 binding and reduced nucleolin association with Lep-2548G/A and Lep-2548A/A, respectively (Fig. 5). The differential binding of Sp1 and nucleolin to Lep-2548G/G and Lep-2548A/A was confirmed by DAPA assays (Fig. $3 b$ ). These results suggested that the presence of Lep-2548A/A and Lep-2548G/ A sequences might increase insulin-dependent activation of the leptin promoter by promoting the exchange between the negative transcriptional regulator nucleolin and the positive regulator Sp1 (Fig. 5). Based on these results, one could speculate that Lep-2548 sequence variations in the leptin gene promoter might contribute to the
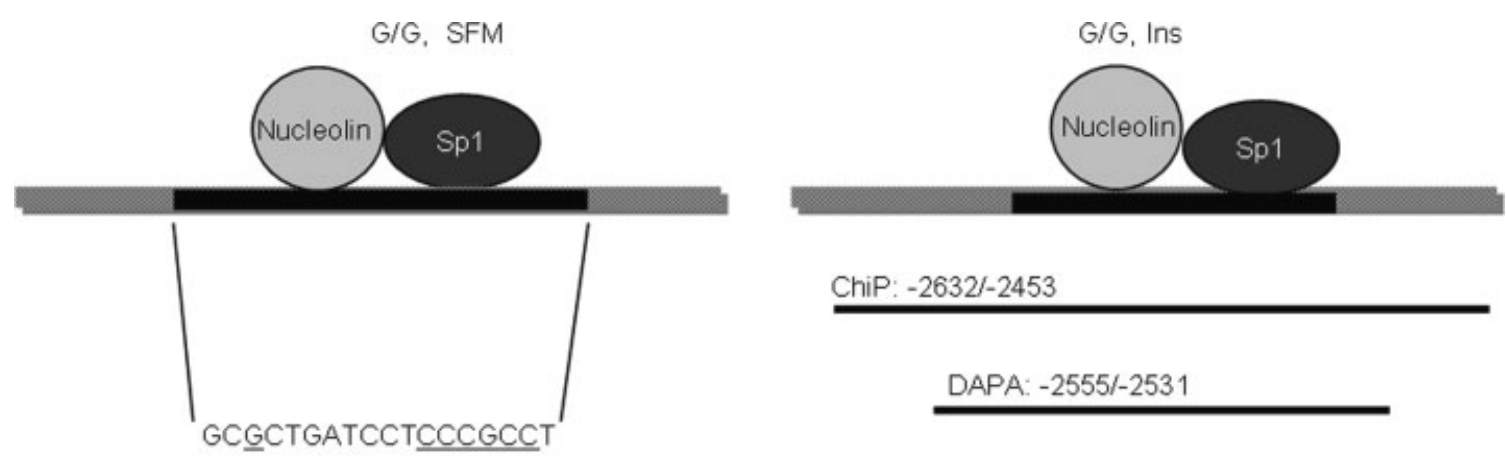

DAPA: $-2555 /-2531$
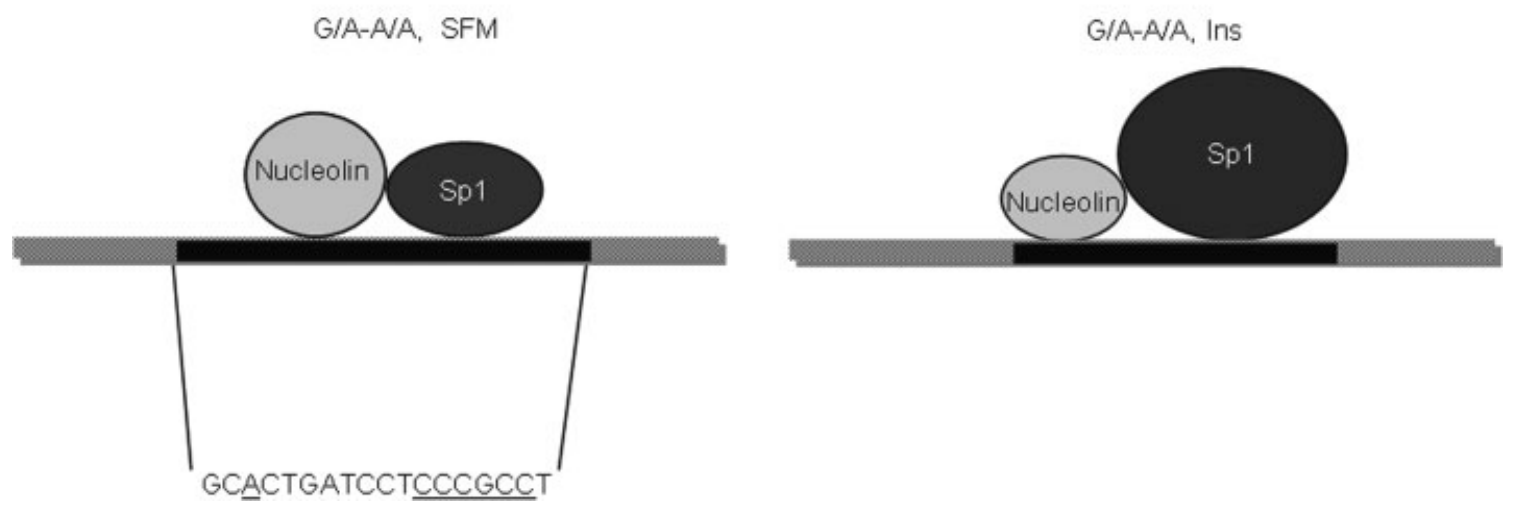

Figure 6 - Model of functional interactions at the Lep-2548 site. Hypothetical model of Sp1/nucleolin binding to the Lep-2548 region in MCF-7, MDA-MB-231 and BT-474 cells untreated (SFM) or treated with insulin (Ins) is presented. The presence of an A allele (Lep-2548G/A, Lep-2548A/A) improves efficient recruitment of Sp1 to DNA under insulin treatment. In contrast, binding of nucleolin to Lep-2548G/A or Lep$2548 \mathrm{~A} / \mathrm{A}$ is downregulated in response to insulin, while it is not regulated on Lep-2548G/G. 
differences in leptin mRNA expression observed in different breast cancer cell lines. Indeed, the lowest levels of basal leptin mRNA were found in MCF-7 cells (Lep-2548G/G), intermediate levels in MDA-MB-231 cells (Lep-2548G/A), and the highest levels in BT-474 cells (Lep-2548A/A) (Fig. 4). Interestingly, MCF-7 cells are characterized by low aggressive, metastatic phenotype, while BT-474 and SK-BR-3 are of a more aggressive phenotype. ${ }^{35}$ Whether or not the expression of leptin in these cells might determine the degree of their malignancy is not yet known.

It is noteworthy, however, that the leptin promoter contains multiple transcription regulatory motifs regulated by insulin. For instance, seven Sp1 sites, and 8 elements binding HIF. ${ }^{22,35,36}$ Our previous study demonstrated that in MDA-MB-231 cells treated with insulin, Sp1 acts through two sites, one of which is in Lep2548 region. On the other hand, functional insulin-regulated Sp1 sites in the leptin promoter have not been mapped in other cells lines. Thus, except for MDA-MB-231 cells, the relative importance of Lep-2548 to basal and insulin-induced leptin expression is difficult to measure in other cells. The possible involvement of multiple transcription mechanisms could explain the very high basal leptin mRNA expression in BT-474 cells and the lack of appreciable insulin-induced stimulation in these cells, despite the apparent activation of the Lep-2548 site. Moreover, it has to be noted that concentrations of insulin used in our study are relatively high (to mimic, at least to some extent) the conditions of hyperinsulinemia. These conditions might lead to activation not only insulin receptors but also receptors for IGF-1 as well as hybrids insulin/ IGF-IR. $^{37,38}$

The presence of Lep-2548G/A and A/A polymorphism in breast cancer cells lines prompted us to investigate whether this genotype can be found in breast tumors. Using LCM, we extracted leptinpositive cancer areas from breast tumors and sequenced DNA of cancer cells (Fig. 6). Interestingly, in all cases, cancer cells contained the Lep-2548A/A polymorphism. The predominance of the Lep-2548A/A genotype might be related to the fact only tumors expressing leptin at high levels were studied. It is possible that more genotypic variability would be detected in greater number of cases characterized by various degrees of leptin expression.

In conclusion, our study suggests that the Lep-2548G/A polymorphism, previously detected in $\mathrm{PBC}$ and associated with increased leptin synthesis, occurs in breast cancer cell DNA and could contribute to leptin overexpression in this cellular context.

\section{Acknowledgements}

This work was supported by the W.W. Smith Charitable Trust and the Sbarro Health Research Organization.

\section{References}

1. Wauters M, Considine RV, Van Gaal LF. Human leptin: from an adipocyte hormone to an endocrine mediator. Eur J Endocrinol 2000; 143:293-311.

2. Han C, Zhang HT, Du L, Liu X, Jing J, Zhao X, Yang X, Tian B. Serum levels of leptin, insulin, and lipids in relation to breast cancer in China. Endocrine 2005;26:19-24.

3. Surmacz E. Obesity hormone leptin: a new target in breast cancer? Breast Cancer Res 2007;9:301.

4. Garofalo C, Surmacz E. Leptin and cancer. J Cell Physiol 2006; 207:12-22.

5. Vona-Davis L, Rose DP. Adipokines as endocrine, paracrine, and autocrine factors in breast cancer risk and progression. Endocr Relat Cancer 2007:14:189-206.

6. Ishikawa M, Kitayama J, Nagawa $\mathrm{H}$. Enhanced expression of leptin and leptin receptor (OB-R) in human breast cancer. Clin Cancer Res 2004:10:4325-31.

7. Koda M, Sulkowska M, Kanczuga-Koda L, Cascio S, Colucci G, Russo A, Surmacz E, Sulkowski S. Expression of the obesity hormone leptin and its receptor correlates with hypoxia-inducible factor-1alpha in human colorectal cancer. Ann Oncol 2007:18 (Suppl 6):vi116-9.

8. Miyoshi Y, Funahashi T, Tanaka S, Taguchi T, Tamaki Y, Shimomura I, Noguchi S. High expression of leptin receptor mRNA in breast cancer tissue predicts poor prognosis for patients with high, but not low, serum leptin levels. Int J Cancer 2006;118:1414-9.

9. Garofalo C, Koda M, Cascio S, Sulkowska M, Kanczuga-Koda L, Golaszewska J, Russo A, Sulkowski S, Surmacz E. Increased expression of leptin and the leptin receptor as a marker of breast cancer progression: possible role of obesity-related stimuli. Clin Cancer Res 2006; 12:1447-53.

10. Zhao L, Shen ZX, Luo HS, Shen L. Possible involvement of leptin and leptin receptor in developing gastric adenocarcinoma. World J Gastroenterol 2005;11:7666-70

11. Yuan SS, Chung YF, Chen HW, Tsai KB, Chang HL, Huang CH, Su $\mathrm{JH}$. Aberrant expression and possible involvement of the leptin receptor in bladder cancer. Urology 2004;63:408-13.

12. Laud K, Gourdou I, Pessemesse L, Peyrat JP, Djiane J. Identification of leptin receptors in human breast cancer: functional activity in the T47-D breast cancer cell line. Mol Cell Endocrinol 2002;188:219-26.

13. Koda M, Sulkowska M, Wincewicz A, Kanczuga-Koda L, Musiatowicz B, Szymanska M, Sulkowski S. Expression of leptin, leptin receptor, and hypoxia-inducible factor 1 alpha in human endometrial cancer. Ann N Y Acad Sci 2007:1095:90-8.

14. Cascio S, Bartella V, Auriemma A, Johannes GJ, Russo A, Giordano A, Surmacz E. Mechanism of leptin expression in breast cancer cells: role of hypoxia-inducible factor-1alpha. Oncogene 2008;27:540-7.

15. Mammes O, Betoulle D, Aubert R, Giraud V, Tuzet S, Petiet A, Colas-Linhart N, Fumeron F. Novel polymorphisms in the $5^{\prime}$ region of the LEP gene: association with leptin levels and response to lowcalorie diet in human obesity. Diabetes 1998;47:487-9.
16. Mammes O, Betoulle D, Aubert R, Herbeth B, Siest G, Fumeron F. Association of the G-2548A polymorphism in the $5^{\prime}$ region of the LEP gene with overweight. Ann Hum Genet 2000;64:391-4.

17. Hoffstedt J, Eriksson P, Mottagui-Tabar S, Arner P. A polymorphism in the leptin promoter region $(-2548 \mathrm{G} / \mathrm{A})$ influences gene expression and adipose tissue secretion of leptin. Horm Metab Res 2002;34: $355-9$.

18. Skibola CF, Holly EA, Forrest MS, Hubbard A, Bracci PM, Skibola DR, Hegedus C, Smith MT. Body mass index, leptin and leptin receptor polymorphisms, and non-hodgkin lymphoma. Cancer Epidemiol Biomarkers Prev 2004;13:779-86.

19. Ribeiro R, Vasconcelos A, Costa S, Pinto D, Morais A, Oliveira J, Lobo F, Lopes C, Medeiros R. Overexpressing leptin genetic polymorphism $(-2548 \mathrm{G} / \mathrm{A})$ is associated with susceptibility to prostate cancer and risk of advanced disease. Prostate 2004;59:268-74.

20. Ribeiro R, Araujo AP, Coelho A, Catarino R, Pinto D, Araujo A, Calcada C, Lopes C, Medeiros R. A functional polymorphism in the promoter region of leptin gene increases susceptibility for non-small cell lung cancer. Eur J Cancer 2006;42:1188-93.

21. Snoussi K, Strosberg AD, Bouaouina N, Ben Ahmed S, Helal AN, Chouchane L. Leptin and leptin receptor polymorphisms are associated with increased risk and poor prognosis of breast carcinoma. BMC Cancer 2006;6:38.

22. Gong DW, Bi S, Pratley RE, Weintraub BD. Genomic structure and promoter analysis of the human obese gene. J Biol Chem 1996; 271:3971-4.

23. Bartella V, Cascio S, Fiorio E, Auriemma A, Russo A, Surmacz E. Insulin-dependent leptin expression in breast cancer cells. Cancer Res 2008;68:4919-27.

24. Tsou JH, Chang KY, Wang WC, Tseng JT, Su WC, Hung LY, Chang WC, Chen BK. Nucleolin regulates c-Jun/Sp1-dependent transcriptional activation of cPLA2alpha in phorbol ester-treated nonsmall cell lung cancer A549 cells. Nucleic Acids Res 2008;36:21727.

25. Sakaguchi M, Miyazaki M, Takaishi M, Sakaguchi Y, Makino E, Kataoka N, Yamada H, Namba M, Huh NH. S100C/A11 is a key mediator of $\mathrm{Ca}(2+)$-induced growth inhibition of human epidermal keratinocytes. J Cell Biol 2003;163:825-35.

26. Ginisty H, Amalric F, Bouvet P. Nucleolin functions in the first step of ribosomal RNA processing. EMBO J 1998;17:1476-86.

27. Erard M, Lakhdar-Ghazal F, Amalric F. Repeat peptide motifs which contain beta-turns and modulate DNA condensation in chromatin. Eur J Biochem 1990;191:19-26.

28. Mongelard F, Bouvet P. Nucleolin: a multiFACeTed protein. Trends Cell Biol 2007:17:80-6.

29. Csermely P, Schnaider T, Cheatham B, Olson MO, Kahn CR. Insulin induces the phosphorylation of nucleolin. A possible mechanism of insulin-induced. RNA efflux from nuclei. J Biol Chem 1993;268: 9747-52. 
30. Morelli C, Garofalo C, Bartucci M, Surmacz E. Estrogen receptoralpha regulates the degradation of insulin receptor substrates 1 and 2 in breast cancer cells. Oncogene 2003;22:4007-16.

31. Morelli C, Garofalo C, Sisci D, del Rincon S, Cascio S, Tu X, Vecchione A, Sauter ER, Miller WH, Jr, Surmacz E. Nuclear insulin receptor substrate 1 interacts with estrogen receptor alpha at ERE promoters. Oncogene 2004;23:7517-26.

32. Cascio S, Bartella V, Garofalo C, Russo A, Giordano A, Surmacz E. Insulin-like growth factor 1 differentially regulates estrogen receptordependent transcription at estrogen response element and AP-1 sites in breast cancer cells. J Biol Chem 2007;282:3498-506.

33. Revillion F, Charlier M, Lhotellier V, Hornez L, Giard S, Baranzelli MC, Djiane J, Peyrat JP. Messenger RNA expression of leptin and leptin receptors and their prognostic value in 322 human primary breast cancers. Clin Cancer Res 2006;12:2088-94.
34. Cascio S, Bartella V, Auriemma A, Johannes GJ, Russo A, Giordano A, Surmacz E. Mechanism of leptin expression in breast cancer cells: role of hypoxia-inducible factor-1alpha. Oncogene 2008;27: $540-7$

35. Clarke RFL, Brunner N, Thompson EW. In vitro model of breast cancer. In: Harris JR, Lippman ML, Morrow M, Hellman S, editors. Diseases of breast. Philadelphia: Lippincott-Raven, 1996.

36. Meissner U, Ostreicher I, Allabauer I, Rascher W, Dotsch J. Synergistic effects of hypoxia and insulin are regulated by different transcriptional elements of the human leptin promoter. Biochem Biophys Res Commun 2003;303:707-12.

37. Belfiore A, Frasca F. IGF and insulin receptor signaling in breast cancer. J Mammary Gland Biol Neoplasia 2008;13:381-406.

38. Pollak M. Insulin and insulin-like growth factor signalling in neoplasia. Nat Rev Cancer 2008;8:915-28. 